\title{
Mínimo vital y salario mínimo, en la política laboral mexicana desde la filosofía política
}

\author{
Vital mínimum and mínimum wage, on Mexican labor policy \\ from political philosophy
}

iD Fernando Cárdenas Cabello

Centro universitario CIFE, México

Correo: cardenascabellofernando@gmail.com

Resumen: El mínimo vital es una concepción que sirve para determinar el nivel más básico de requerimientos de los cuales se vale una persona para su subsistencia y satisfacer sus necesidades más apremiantes, entendidas así desde la perspectiva de los derechos humanos. El salario mínimo en México, determinado por una comisión tripartita tendría que contemplar estos aspectos para recuperar el poder adquisitivo que tal elemento componente de los derechos laborales ha perdido en México y que este (el salario mínimo) deje de servir sólo como un indicador para la imposición de multas y referente de empleos infra retribuidos, para recuperar lo que a mediados del siglo pasado fue, un parámetro de retribución equitativa al trabajo, que permita la subsistencia digna de la persona. Después de una revisión comparativa del salario mínimo en México con el de otros países de Latinoamérica, se identificó que este indicador en México es de los más bajos es esta región, se concluye que la adecuación y aumento del salario mínimo en México, si bien ajustada en la actual administración federal, resulta necesario que se siga aumentando y se hace una propuesta.

Palabras clave: justicia social; mínimo vital; política laboral; salario mínimo

\begin{abstract}
Vital Minimum is a concept used to determinate the lowest requirements level which a person uses for it own subsistence and to satisfy their most basics needs, understand so, from the human rights perspective. Minimum wage on México, determined by a tripartite commission should contemplate this elements to recuperate the purchasing power that this component of labor law has lost in México, and this (minimum wage) stops working only as an indicator to impose fines and a parameter for bad remunerated employments, to recover whatever once it was at the middle of the past century, an equitable parameter of retribution of work that allows dignity subsistence for the person. After a comparative revision of minimum wage in México with other Latin America countries, was identified that this indicator in México is one of the lowest on this region, is concluded that an adaptation and increase of minimum wage on México, adjusted on current federal administration, results necessary it stills increasing and a proposal is made.
\end{abstract}

Keywords: labor policy; minimum wage; social justice; vital minimum

Cárdenas-Cabello, F. (2021). Mínimo vital y salario mínimo, en la política laboral mexicana desde la filosofía política. [Vital mínimum and mínimum wage, on Mexican labor policy from political philosophy].

Forhum International Journal of Social Sciences and Humanities, 3(4), 97-107. https://doi.org/10.35766/j.forhum2021.04.03.8

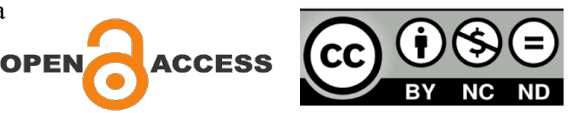




\section{Introducción}

El mínimo vital es un constructo conceptual que parte de la idea de otorgar a la persona trabajadora medios de subsistencia suficientes para el desarrollo de una vida digna en una sociedad democrática, este concepto está presente en las más fundamentales declaraciones internacionales (Declaración Universal de los Derechos Humanos [DUDH] art. 23), en los preceptos esenciales de diversos países como México (Constitución Política de los Estados Unidos Mexicanos [CPEUM]) y en las directrices de instituciones de orden internacional (Organización Internacional del Trabajo [OIT]) y nacional en México (Comisión Nacional de los Derechos Humanos [CNDH]).

Este trabajo busca argumentar la importancia del salario mínimo en tanto que materialización del mínimo vital, desde la óptica de la filosofía política contemporánea y uno de sus conceptos centrales, la justicia social; entendida como el acceso a los bienes sociales primarios, según lo planteó Rawls (2014) en la segunda mitad del siglo pasado y lo han recuperado autores como Pogge (2008) y Sen (2010) en la presente centuria.

El cuestionamiento y objetivo al cual responde este estudio es ¿cómo tendría que actualizarse el salario mínimo en México para poder alcanzar con este indicador el acceso al mínimo vital en este país? Considerando antecedentes y parámetros tanto históricos (de México), como actuales exógenos, en comparación con otros países de Latinoamérica. Para alcanzar las metas de argumentar la necesidad de revalorizar el salario mínimo en México en su dimensión histórica como en comparación de otras naciones latinoamericanas, se recurre a los estudios más actualizados sobre el salario mínimo en México (Campos \& Rodas, 2020; Guerrero \& Lomelí, 2017; Moreno-Brid et. al., 2014), de la política laboral mexicana (Alzaga, 2006), el mínimo vital (Carmona, 2012) y la justicia social (D'Amico, 2015); desde una perspectiva transdisciplinar con la ayuda de trabajos desde la economía, el derecho, y las ciencias sociales (Bialakowsky \& Hermo, 2015) y políticas (Pérez, 2017).

\section{Metodología}

Se buscaron referencias en las bases de datos de Elsevier y Mendeley, bajo los motores de búsqueda: "salario mínimo en México" y "justicia social en México", se depuró a resultados de documentos de las ciencias sociales y se privilegiaron los trabajos más recientes. Se efectuó el estudio del objeto a través de los métodos histórico y comparativo (Grawitz, 1975). El método histórico que observa un objeto a través del tiempo, pues se estudia la cuantía del salario mínimo en México durante la segunda mitad del siglo XX y lo que va del XXI. Y el método comparativo, que analiza un mismo fenómeno en distintas latitudes, dado que se contrasta la cuantía actual del mismo indicador (salario mínimo) en varios países de América Latina.

\section{Resultados}

\section{Mínimo vital y salario mínimo}

Mínimo vital

En la Declaración Universal de los Derechos Humanos (Organización de las Naciones Unidas [ONU], 1948), se estableció en su artículo 25: 
Toda persona tiene derecho a un nivel de vida adecuado que le asegure, así como a su familia, la salud y el bienestar, y en especial la alimentación, el vestido, la vivienda, la asistencia médica y los servicios sociales necesarios. (p.7).

El citado artículo, junto con los anteriores (del 22 al 25), de la Declaración, pueden agruparse bajo la denominación general de derechos de los trabajadores. El fragmento reproducido nos ayuda a comprender qué es lo que debemos entender por mínimo vital, que no es otra cosa que el acceso a bienes de subsistencia en su mínima expresión, pero que permitan a la persona, en este caso al trabajador y quienes económicamente de él dependen, el acceso a una vida digna, alejada de las carencias y la inmundicia.

El rastreo de diferentes concepciones de medios mínimos de subsistencia, como un derecho del ciudadano, puede efectuarse en diferentes etapas de la historia de occidente, a partir del imperio romano (Carmona, 2012), durante la edad media y en el siglo XX, especialmente en el arribo de los estados de economía centralizada, aunque por supuesto también en los de economía de libre mercado.

El mínimo vital concebido como un derecho puede entenderse así según Carmona (2012):

La libre disposición de unos recursos económicos mínimos para hacer frente a las necesidades más perentorias del ser humano... estos recursos pueden provenir bien del pago de un salario a los trabajadores... o bien de una cantidad periódica abonada por los poderes públicos a aquellos ciudadanos que no realizan un trabajo remunerado (parados, ancianos, enfermos, discapacitados, etc.). (p. 1579).

El derecho al mínimo vital se encuentra presente en la Constitución Política de los Estados Unidos Mexicanos (CPEUM), no estrictamente de manera textual, pero sí comprendido en la esencia de varios artículos, según la Suprema Corte de Justicia (México, Suprema Corte de Justicia de la Nación [SCJN], 2013):

En el orden constitucional mexicano, el derecho al "mínimo vital"... cobra vigencia a partir de

la interpretación sistemática de los derechos fundamentales consagrados en la Constitución

Política de los Estados Unidos Mexicanos, particularmente en sus artículos 1o., 3o., 4o., 13, 25 , 27, 31, fracción IV, y 123. (p.1)

Como se ha expuesto, el mínimo vital en su materialización u operativización por parte del Estado, presenta dos formas: la asistencia social como dotación de recursos a los no trabajadores, y el salario mínimo en tanto que retribución al trabajo realizado. Pasemos a tratar esta segunda forma, que es a la que nos limitaremos en este estudio.

\section{Salario mínimo}

En la Declaración Universal de los Derechos Humanos de 1948, encontramos una clara alusión al salario mínimo, en el párrafo 3 del artículo 23, el cual dice: "Toda persona que trabaja tiene derecho a una remuneración equitativa y satisfactoria, que le asegure, así como a su familia, una existencia conforme a la dignidad humana" (ONU, 1948, p. 7).

La Organización Internacional del Trabajo lo define así ([OIT], 2014, párrafo 68): "la cuantía mínima de la remuneración que un empleador deberá abonar a sus asalariados por las prestaciones que éstos hayan efectuado durante un determinado período, sin que dicha cuantía pueda ser rebajada mediante convenio colectivo ni acuerdo individual".

En México la existencia del salario mínimo, como intento de reflejo del mínimo vital está presente desde el texto constitucional, en el Art. 123, apartado A, fracción VI, añadido en 1961 a iniciativa del entonces presidente Adolfo López Mateos, del siguiente modo: 
Los salarios mínimos que deberán disfrutar los trabajadores serán generales o profesionales... Los salarios mínimos generales deberán ser suficientes para satisfacer las necesidades normales de un jefe de familia, en el orden material, social y cultural, y para proveer a la educación obligatoria de los hijos. Los salarios mínimos profesionales se fijarán considerando, además, las condiciones de las distintas actividades económicas.

El salario mínimo, en tanto que noción presente en el máximo ordenamiento jurídico de la nación, busca también responder a la necesidad de la preservación de los derechos humanos de la ciudadanía mexicana, la garantía de los derechos humanos está presente en diversos tratados internacionales de los cuales México es signatario. Por desgracia, existen algunas formas de trabajo, que no son cubiertas por el derecho al salario mínimo, ni los derechos sociales en general, como el retiro, la pensión o incluso la remuneración; como el trabajo doméstico (no de empleados domésticos, sino de amas de casa) y el voluntariado; y otras formas emergentes de trabajo como el teletrabajo por vías electrónicas y aquellos en los cuales no es fácil establecer ni responsabilizar al empleador por una relación laboral, como algunas plataformas digitales de diferentes giros tales como Didi, Uber, Uber Eats.

Para la república Mexicana, la determinación de la cifra exacta del salario mínimo es resultado de las negociaciones de la Comisión Nacional de Salarios Mínimos (CONSAMI), organismo en el cual intervienen representantes del sector trabajador, patronal y gubernamental; cuya existencia tiene fundamento en el mismo artículo 125 constitucional, apartado A, fracción VI. Actualmente en México existe una profunda problemática en la determinación de la cuantía del salario mínimo mensual -por mencionar un ejemplo claro- en marzo de 2016 (México, CNDH, s.f.), este ascendió a \$ 70.10 diarios; cantidad multiplicada por 31 (días que en caso máximo tiene un mes) resultó en $\$ 2,173.1$. Mientras que el mismo mes, la línea de bienestar mínimo (Indicador económico que contempla el costo monetario de una canasta básica de alimentos, mas algunos otros bienes y servicios), tenía un costo de 2,714.66. Así, como se puede observar con claridad, el salario mínimo era insuficiente para cubrir la línea de bienestar.

Otro problema, radica en la homologación que se hizo en 2015 en un solo monto para todas las zonas económicas del país, pues el costo de la vida es muy variable entre las diferentes entidades federativas e incluso al interior de las mismas, según la ciudad de que se trate. Antes de 2013, las zonas económicas en que se dividía el territorio nacional eran A, B y C; en 2015 sólo A y B; y en 2015 una sola para todo el país con un mismo monto para el salario mínimo. Debe hacerse notar en este espacio la iniciativa de la administración federal 2018-2024, de revalorizar el poder adquisitivo del salario mínimo, que en 2019 lo aumentó y lo dividió en dos zonas, la fronteriza con U.S.A. (Zona Libre de la Frontera Norte) y la no fronteriza, o resto del país; y para 2020 lo actualizó (México, Secretaría del Trabajo y Previsión Social [STPS], 2019) para quedar en \$185.56 diarios en la primera, y en $\$ 123.22$ en la segunda. Una valoración de la actualización cronológica del salario mínimo en México se efectuará en la parte III de este trabajo.

\section{Política laboral y Justicia social Política laboral}

La política laboral mexicana engloba las acciones del gobierno, tendientes a la regulación de las relaciones entre las fuerzas productivas del país, básicamente patrones y trabajadores y sus respectivas representaciones, para esta regulación se vale de instrumentos legales e institucionales, la referencia legal de más alta jerarquía la encontramos en la propia CPEUM, precisamente en el art. 
123, y en la propia Ley Federal del Trabajo de México; mientras que institucionalmente en el ámbito federal el referente principal es la Secretaría del Trabajo y Previsión Social. El artículo 123 constitucional regula de manera general las relaciones laborales entre el sector patronal y sus trabajadores, en el apartado A; mientras que en el apartado B se aboca al propio gobierno federal como empleador, con algunos elementos que atañen a los poderes locales, como la fracción IV, párrafo segundo.

La STPS en tanto que institución encargada de la materialización de la política laboral en encuentra la descripción de sus funciones principales en el artículo 40 de la Ley Orgánica de la Administración Pública Federal, mientras que la especificación de la estructura para su funcionamiento queda plasmada en el Reglamento interno de la STPS, Alzaga (2006, p. 143) condensa los principios de la política laboral en México en los siguientes: "1. El empleo, 2. El salario, 3. El reconocimiento y aplicación de los derechos básicos laborales e impartir justicia laboral. 4. Los conflictos obreros-patronales, sobre todo, los colectivos y 5. La política sindical". Sobre la política laboral en su conjunto, en tanto que obligación del Estado, se observa (Alzaga, 2006) que ha presentado un retroceso o debilidad en México durante las últimas administraciones federales, pero especialmente en la primera del presente milenio. Tendencia mantenida en las dos subsecuentes y que en la iniciada en 2018, en algunos elementos como la ratificación de convenios internacionales y la regulación del outsourcing se ha buscado disminuir.

\section{Justicia social}

El concepto, es un tema contemporáneo de debate en las ciencias sociales del siglo XX y XXI, y es motivo para la transversalidad disciplinaria entre tradiciones académicas como la filosofía, el derecho, la economía y la política. La filosofía, ha sido la iniciadora en términos teóricos, por su parte la ciencia jurídica aporta al tema, incluso con anterioridad, al disponer con la emisión de reglas, directrices en la búsqueda de la materialización del ideal de una sociedad justa.

En cuanto a la aportación del derecho a la construcción del concepto, es posible encontrar en los propios principios fundacionales de la OIT, de manera concreta en el preámbulo de su constitución que data de 1919, la alusión a la justicia social, sobre la cual se afirma que la paz universal y permanente sólo puede fundarse en la justicia social. Por su parte en el caso particular de México podemos ubicar la noción de justicia social en las constituciones de 1824, 1857, pero especialmente en la de 1917. La ley, en términos generales busca la materialización de la justicia, y no sólo entre los individuos con las diversas materias del derecho, sino, valdría decir ente las clases y sectores sociales.

La justicia social, desde la perspectiva de la filosofía y la política, es uno de los debates centrales actuales en ambas disciplinas; desde que centrara su mirada en el tema Rawls (2014) en 1971 con la publicación de su ya clásico estudio en la materia Teoría de la justicia, en el contexto aún de la guerra fría; en ese extenso tratado pone de manifiesto la irrenunciable obligación del Estado de proveer al ciudadano de una base mínima de beneficios, englobados por él bajo la denominación de bienes sociales primarios, consistentes en: derechos, libertades, oportunidades, autorrespeto, y riqueza.

El estudio parte de la crítica al utilitarismo presente en la sociedad contemporánea, que privilegia la obtención maximizada de beneficios aún en detrimento de los bienes más básicos de otros miembros de la colectividad. Para argumentar su teoría ofrece dos principios: el de libertades y el de obligaciones. El primero radica en que todos los miembros de la sociedad posean un esquema básico mínimo de libertades irrenunciables. Mientras que el segundo se refiere a que si el Estado ha de actuar diferenciadamente con algunos sectores sociales para favorecerlos, ha de ser precisamente 
sobre los cuales no poseen las capacidades mínimas para allegarse por sí solos de los bienes sociales primarios.

En el planteamiento de su teoría, Rawls acuña algunos conceptos que son clave para comprenderla a cabalidad como: la posición original, que es el momento hipotético de la celebración del contrato social en el que se definen las reglas de operación de la vida social, así como las retribuciones al interior de la misma; otro de estos conceptos es el de velo de la ignorancia, que se refiere al desconocimiento del actor social del resultado que tendrá sobre sí y su lugar de ventaja o desventaja en la sociedad, con la aplicación de las reglas establecidas en la posición original.

Estos postulados sobre la recuperación de la importancia de la ética y la moral en la reflexión política, por oposición al conductismo y la elección racional, debates que definieron los estudios de la disciplina durante el siglo XX; son continuados por Amartya Sen, laureado con el Nobel en economía por sus estudios sobre las hambrunas, él mismo se asume como continuador del pensamiento rawlsiano y le otorga a este pensador un lugar central en el pensamiento del siglo XX, aunque se desprende de Rawls al observar que la búsqueda de la justicia actualmente, no puede circunscribirse a una escala nacional (Sen, 2010).

El anhelo de la materialización de la justicia como un objetivo internacional, parte de la idea de que el sistema mundial es injusto, lo cual se ha denunciado con claridad al señalar la realidad presente, en la que los capitales internacionales son capaces de evadir las reglas que mucho tiempo le llevaron construir a la organización estatal nacional: evaden impuestos, trasgreden derechos, desestabilizan gobiernos y sociedades, degradan el ambiente; todo ello en detrimento de personas y pueblos enteros, en beneficio y concentración de muy pocos. Ante tal situación surge la idea de la materialización de la justicia global como ideal contemporáneo, de la mano de autores como el comentado Sen (2010) y otros como Pogge (2008), quien añade al debate elementos contundentes como las cifras de hambruna en regiones como África en pleno siglo XXI en contraste con la opulencia y dispendio de regiones como Europa y la Norteamérica anglosajona; por lo cual es urgente la reforma del orden internacional global que atienda a estas problemáticas.

Hoy la democracia debe ser entendida más allá de la mera representación política o participación en la elección de los gobernantes, en la actualidad debe contemplar la ampliación de derechos y un profundo alcance de los mismos que permitan al ciudadano gozar de libertades no sólo políticas sino económicas, así, los Estados contemporáneos (Pérez, 2017), en un afán de promover estas libertades económicas deben practicar una política social que permita a los ciudadanos el acceso a los bienes sociales primarios. Los programas de desarrollo social (D'Amico, 2015), la promoción del empleo y la regulación del mismo, así como su equitativa retribución a través del salario; las cuales son parte, entre otras, de estas obligaciones sociales del Estado democrático contemporáneo.

Desde una perspectiva latinoamericana y por añadidura mexicana, el concepto de justicia social ha sido recuperado, especialmente ante una realidad en la que la globalización y la implementación de los preceptos económicos neoliberales en la región (Bialakowsky \& Hiermo, 2015) han agudizado las de por si precarias condiciones económicas de las respectivas sociedades nacionales, no solamente en el aspecto laboral, también en lo relativo a la salud y al acceso a la educación de calidad. La justicia social pues se presenta como una necesidad ante un contexto de marcada desigualdad social, la retribución que busque permitir el acceso al mínimo vital es una parte importante en la búsqueda de esa justicia social. 


\section{Comparativa entre el salario mínimo en histórico México y actualmente en América Latina El salario mínimo en México desde mediados del siglo XX}

Moreno-Brid et. al. (2014), dividen el desempeño del salario mínimo en México en tres periodos de evolución, a partir de la década del 50 del siglo pasado, que se relacionan con la dinámica de la propia la economía nacional: el primero que va de la década de 1950 hasta mediados de la de 1970, cuando el salario mínimo se cuadriplicó, mientras la economía crecía en el orden de $6 \%$ en el PIB con una ligera inflación; el segundo desde alrededor de 1977 hasta alrededor de 1995, en este último año el salario mínimo había perdido el $66 \%$ del poder adquisitivo con respecto de 1980 , este periodo se caracterizó por una alta tasa inflacionaria y una baja actividad económica; el tercer periodo a partir del mismo 1995 y hasta 2014 (cuando se cierra su estudio), entonces el salario mínimo, mantuvo su depreciación, aunque a un nivel menor que en el periodo previo, una inflación contenida y un dinamismo económico constante aunque no en las cifras de mediados del siglo pasado.

En el mismo año de 2014, se dio a conocer un documento (Guerrero \& Lomelí, 2017) por parte del gobierno del entonces Distrito Federal (la capital del país), elaborado por un grupo de expertos, quienes propusieron una apreciación del salario mínimo en esa entidad de la federación, la cual fue instrumentada por el gobierno de tal localidad; con lo cual el monto diario del salario pasó de \$ 67.29 a \$ 82.86. En réplica el gobierno federal actualizó a razón de un $4 \%$ anual el salario mínimo nacional entre 2014 y 2016, mientras que en un porcentaje de alrededor de $10 \%$ los dos años posteriores. La administración federal mexicana que inició su ejercicio en 2018 aumentó el salario mínimo en un $16.21 \%$ para 2019 , y en un $20 \%$ para 2020 , para quedar en $\$ 123.22$ diarios este último año (México, STPS, 2019).

Con el aumento porcentual del salario mínimo por la actual administración federal mexicana, observamos un visible afán por revalorizar el trabajo asalariado, y que su retribución constituya una mejor herramienta para solventar los requerimientos de subsistencia de la población. Como se puede observar la evolución de la actual cuantía del salario mínimo en México, ha recuperado poder adquisitivo relativo en comparación al promovido por inmediatas administraciones federales pasadas, al incrementarse en cifras superiores a las de la inflación. Revisemos ahora el valor del poder adquisitivo del salario mínimo mexicano en comparación con el de otros países latinoamericanos.

Salario mínimo mexicano en 2020 y su comparación con salarios mínimos de países latinoamericanos

Un parámetro de comparación equitativo para el salario mínimo mexicano debe ser con respecto de otra sociedad en la que el costo de la vida sea similar, por ello se plantean como punto de comparación para esta reflexión algunos países latinoamericanos. Moreno-Brid et. al. (2014) señalan que el salario mínimo en México no sólo es de los más bajos en América Latina, también es de los de menor poder adquisitivo; ello a pesar de que la productividad laboral media en México para 2011, era de las más altas de la región, sólo por debajo de la de Chile.

En una nota de 2016, la British Broadcasting Company (BBC), con datos de la consultora internacional MoveHub (Justo, 2016), agrupó ciertos países latinoamericanos, en los cuales la canasta básica tiene un similar costo proporcional, con respecto del total del salario medio (entre el $10 \mathrm{y}$ el $20 \%$ ), en el mismo grupo de países encontramos a México, Uruguay, Venezuela, Chile, Argentina y 
Cuba (el estudio señala tergiversación en las cifras de inflación emitidas por los gobiernos de Venezuela y Argentina). No pasa desapercibido que se aglutine en el mismo grupo a economías tan disímbolas como Chile y Venezuela o Cuba, pero recordemos que el parámetro no es en términos puros sino proporcionales.

En la gráfica 1, observamos la cuantía del salario mínimo en dólares americanos de algunos países latinoamericanos, según un artículo de la cadena americana Cable News Network ([CNN] 2019). A la cual se recurre para, una vez establecidos parámetros de similares economías, observar la cuantía respectiva en dólares americanos de tales economías y a partir de ello intentar determinar la cuantía que el salario mínimo en México tendría que alcanzar.

\section{Gráfica 1: Salario mínimo en dólares americanos de algunos países de América Latina (2019)}

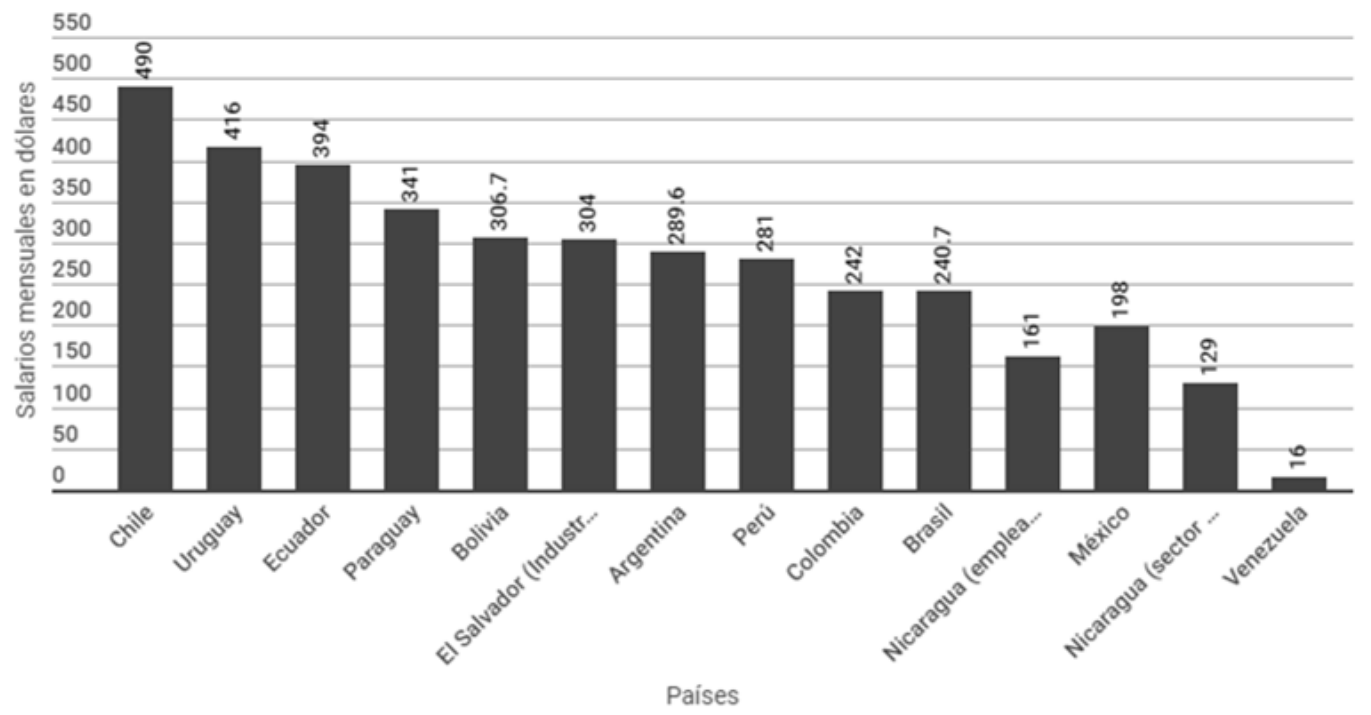

Fuente: CNN Español (17 de diciembre, 2019).

Con los parámetros anteriormente mencionados, para que el poder adquisitivo del salario mínimo en México se equipare al de similares sociedades en la misma región, tendría que elevarse en cuantía total en dólares americanos, al de Chile y Uruguay, los más altos en la región, en un escenario óptimo; o bien al de Argentina, Colombia o Brasil, en el peor de los escenarios. Se propone como equitativo, comparar el salario mínimo "para el resto del país", con el de menor cuantía de los países listados. Así, el día de hoy (19 de noviembre de 2020 con un tipo de cambio de \$20.32 pesos mexicanos por dólar americano) el salario mínimo en Brasil (el país de los mencionados donde el salario mínimo es más bajo) en pesos mexicanos, asciende a los 4891.024. Diferente y superior en más de $\$ 1,000$ pesos mexicanos, a los 3,819.82 que se pagan en este último país como salario mínimo.

Si se prevé un ajuste para el 2021 en el salario mínimo en México, este sería previsiblemente congruente al más reciente, que fue de 20\%; con lo cual podría ascender a: \$ 147.864 diarios, lo que al mes (31 días) resulta en: \$ 4583.784, con ese ajuste sigue por debajo de su similar en Brasil, y esto sin contar los aumentos que tuvo el salario mínimo en el país carioca en 2020 y el que podría tener para 2021. 
Se presenta como un parámetro equitativo el comparar el salario mínimo fronterizo Zona Libre de la Frontera Norte (ZLFN)", con el del país límite superior de los comparados comentados, que es Chile. Así el salario mínimo mensual ZLFN de México en 2020 fue de \$5,752.36. Mientras que en Chile ya convertido a moneda mexicana (en 2019) fue de $\$ 9,956.8$. En esta comparación el resultado es notablemente divergente. No es posible ignorar que el ajuste que el salario mínimo sufrió en Chile en el 2019, surgió como una respuesta del gobierno de Piñeira a las manifestaciones sociales de ese momento (CNN, 2019).

\section{Discusión y conclusiones}

El mínimo vital es un constructo conceptual de la filosofía social, el cual constituye la esencia del salario mínimo, mismo que se materializa como un indicador tangible y ponderable numéricamente, que a la vez refleja la política laboral en un país de un determinado gobierno en turno, así como la disposición del mismo para materializar en la práctica uno de los objetivos del estado democrático contemporáneo, la justicia social. En este sentido la tradición iniciada por Rawls (2014) y continuada por Sen (2010) es continuada por Pogge (2008) y los trabajos de Bialakowsky \& Hiermo, 2015 y D'Amico, 2015, contribuyen al tratamiento del tema de la justicia social en América Latina, mientras que Pérez (2017) puntualiza estos retos para el Estado mexicano, de materializar la justicia social como requisito imprescindible para considerarse como democrático. La valorización y retribución del trabajo de un amplio grupo de la sociedad a través del aumento del salario mínimo en México, es una exigencia actual de la materialización de la mencionada justicia social.

Existe una noción de la economía, defendida incluso hacia el final del siglo pasado, que plantea que si se aumenta el salario se desincentiva la contratación, la cual ha sido refutada experimentalmente por Card \& Kruegel (citados por Campos, 2015). Uno de los principales economistas especialistas en salario mínimo en México, Campos afirma categóricamente: "Cuando el incremento del salario mínimo es "modesto" y se tiene un nivel bajo de antemano los efectos de incrementar el salario mínimo en empleo son nulos" (Campos, 2015, p. 102). De modo que el gobierno mexicano no tendría por qué temer -como se ha visto en los dos últimos años-en aumentar, aunque sí gradualmente, el salario mínimo. Lo cual es positivo para la economía familiar en general, pues una buena valorización del salario mínimo suele redundar en disminución de pobreza laboral y mejora de ingresos salariales, lo que los especialistas denominan, efecto faro (Campos \& Rodas, 2020).

Proponer una cifra precisa de la cuantía en que tendría que fijarse el salario mínimo en México el día de hoy (para el año 2021) para cubrir el mínimo vital es muy arriesgado. Sin embargo, sí es posible aventurarnos a ofrecer un rango. Ello con fundamento en el costo de la canasta básica en comparación con otros países de la región. Así, el rango de la cifra del salario mínimo en México tendría que ser, entre la compensación que se ofrece en Brasil como parámetro menor, y la que se ofrece Chile como parámetro superior, para el tipificado como ZLFN. Una vez que se homologue con el de sociedades con similar costo y poder adquisitivo, el salario mínimo debería de actualizarse según el índice nacional de precios al consumidor, para que conserve la capacidad de permitir al trabajador el acceso al mínimo vital como derecho humano.

En el actual gobierno federal mexicano 2018-2024, se han hecho importantes esfuerzos por revalorizar el salario mínimo en México, pero este sigue siendo uno de los más bajos de Latinoamérica, por desgracia ante la inminente recesión económica que se avecina por efecto de la 
pandemia por COVID-19 (Cárdenas-Cabello \& Luna-Nemecio, 2020; Luna-Nemecio, 2020), tal vez este esfuerzo de aumento en el monto se vea detenido, pero urge que la actualización se mantenga constante, para equipararse con el de economías similares en América Latina. Al igual que los salarios en general en el país.

\section{Referencias}

Alzaga, O. (2006). La política laboral en México y la pretendida reforma laboral. ALEGATOS, 62, 141-158. http://alegatos.azc.uam.mx/index.php/ra/article/view/513/501

Bialakowsky, A. \& Hermo, J. (2015). Repensar la sociología del trabajo desde el Sur Global Nuevos y viejos desafíos para comprender los procesos sociales de trabajo en el capitalismo globalizado. Revista mexicana de ciencias políticas y sociales, 60 (224), 45-70. DOI: 10.1016/S0185-1918(15)30003-9

Cable News Network [CNN]. (17 de diciembre de 2019). Los salarios mínimos más altos (y bajos) de América Latina en 2019. https://cnn.it/3suc7bg

Campos, R. (2015). El salario mínimo y el empleo: Evidencia internacional y posibles impactos para el caso mexicano. Economía, 12(36), 90-106. DOI: 10.1016/j.eunam.2015.10.006

Campos, R. \& Rodas, J. (2020). El efecto faro del salario mínimo en la estructura salarial: evidencias para México. El trimestre económico, 87(345), 51-97. https://doi.org/10.20430/ete.v87i345.859

Cárdenas-Cabello, F. \& Luna-Nemecio, J. (2020). Evaluación en línea ante la pandemia por Covid19: retos y oportunidades para las universidades mexicanas. Universidad y Sociedad, 12(6), 393-403. https://rus.ucf.edu.cu/index.php/rus/article/view/1860

Carmona, E. (2012). El derecho a un mínimo vital. Derechos sociales y tutela antidiscriminatoria, Aranzadi, 1577-1616. https://archivos.juridicas.unam.mx/www/bjv/libros/11/5154/25.pdf

D'Amico, V. (2015). De la pobreza a la desigualdad. Discursos internacionales, efectos nacionales. Latinoamérica, 61, 237-263. DOI: 10.1016/j.larev.2015.12.010

Grawitz, M. (1975). Métodos y técnicas de las ciencias sociales. Hispano Europea.

Guerrero, C. \& Lomelí, L. (2017). Reflexiones teóricas en torno a la propuesta de recuperación del poder de compra del salario mínimo en México. $C y$, 64-1, 958-971. http://www.cya.unam.mx/index.php/cya/article/view/1009/914

Justo, M. (26 de febrero de 2016). ¿Qué países tienen la canasta básica más cara (y barata) en América Latina? BBC mundo. https://bbc.in/39BxwXh

Luna-Nemecio, J. (2020). Determinaciones socioambientales del COVID-19 y vulnerabilidad económica, espacial y sanitario-institucional. Revista de Ciencias Sociales,26(2), 21-26. http://dx.doi.org/10.31876/rcs.v26i2.32419

México. (1917). Constitución Política de los Estados Unidos Mexicanos.

México. Secretaría del Trabajo y Previsión social [STPS]. (2019). https://bit.ly/3ssWscb

México. Suprema Corte de Justicia de la Nación [SCJN]. (2013). Derecho al mínimo vital. Concepto, alcances e interpretación por el juzgador. Semanario Judicial de la Federación y su Gaceta. https://sjf.scjn.gob.mx/sjfsist/Documentos/Tesis/2002/2002743.pdf

Moreno-Brid, J., Garry, S. \& Monroy, L. (2014). El salario mínimo en México. ECONOMÍAunam, 11(33), 78-93. http://www.revistas.unam.mx/index.php/ecu/article/view/47075/42379

Organización de las Naciones Unidas [ONU]. (1948). Declaración universal de los derechos humanos. https://www.ohchr.org/EN/UDHR/Documents/UDHR_Translations/spn.pdf 
Organización Internacional del Trabajo [OIT]. (2014). Sistemas de salarios mínimos, Estudio General. https://www.ilo.org/global/standards/WCMS_235305/lang--es/index.htm

Pérez, G. (2017). Los desafíos sociales de la democracia en México. Estudios politicos, 41, 27-53. DOI: $10.1016 /$ j.espol.2017.02.001

Pogge, T. (2008) ¿Qué es la justicia global? Revista de Economía Institucional, 10(19), 99-114. https://www.redalyc.org/pdf/419/41901905.pdf

Rawls, J. (2014). Teoría de la justicia. Fondo de Cultura Económica.

Sen, Amartya. (2010). La idea de la justicia. Random House. 